\title{
Análisis histórico de la profesión de recursos humanos en Puerto Rico desde 1960 al 2000
}

\author{
Ángel Rivera Aponte / alra71@yahoo.com \\ Universidad de Puerto Rico, Recinto de Río Piedras \\ Françoise Latortue Albino / francoise.latortue@outlook.com
}

Recibido: 24 de mayo de 2012

Aceptado: 15 de febrero de 2013

\section{RESUMEN:}

Esta investigación examina el comportamiento de la fuerza laboral en la profesión de recursos humanos desde 1960 al 2000 en Puerto Rico. Se estudiaron las tendencias y el desarrollo de dicha fuerza laboral por género, estructura ocupacional, brecha salarial y cantidad de ofrecimientos académicos a nivel de educación superior. Los resultados del análisis demuestran que la profesión continúa en crecimiento entre ejecutivos, administrativos y gerentes, y sugieren la necesidad de promover estudios relacionados con la especialidad, así como determinar los factores que inciden en la brecha salarial, y generar estrategias de integración entre la oferta y la demanda.

Palabras claves: profesión de recursos humanos, planificación de recursos humanos, género, brecha salarial, oferta académica

\section{ABSTRACT:}

This investigation examines the labor force behavior in human resources career between 1960 through 2000 in Puerto Rico. The study includes tendencies of the labor force by gender, occupational systems, gap wage salary, and the quantity of academic programs offered in the higher education level. The results of the analysis demonstrate that the human resource field is in continuous development between executives, administrator and managers, and suggest the need of promoting studies related to major as well as to determine gender gap salary factors, and bring integration strategies between demand and offer.

Keywords: profession of human resources, human resources planning, gender, gap wage salary, academic programs offered

\section{INTRODUCCIÓN}

La planificación de recursos humanos es una de las funciones fundamentales dentro de la profesión de los recursos humanos. Uno de los componentes que se estudia en la planificación de recursos 
humanos es el comportamiento de la fuerza laboral. El concepto de planificación de fuerza laboral fue desarrollado en los años 60. Este implica estimar cómo será en el futuro la actual fuerza laboral, determinar la fuerza laboral requerida para el futuro e identificar posibles espacios y oportunidades de empleo. La planificación de fuerza laboral se valió de los modelos matemáticos para cuantificar el suministro y la demanda de la misma. Este concepto técnico tenía un fuerte enfoque cuantitativo, y de modo general se utilizaba sin considerar problemas más amplios, en consecuencia, fue removido de los procesos de administración de la empresa (Director, 1985).

La planificación de fuerza laboral comenzó a asumir un rol más amplio a finales de los años 70 y se vinculó a la planificación estratégica y operacional, pero todavía se enfocaba en modelos de pronóstico que no tomaban en cuenta factores como ambiente político y social, condiciones organizacionales y posibles cambios en la dirección corporativa (Hopkins, 1988). En ese momento, la teoría y práctica de manejo de la planificación de los recursos humanos comenzó a reconocer a los empleados como una fuente y no solo como un gasto. La confusión emergió, sin embargo, al considerarse hasta dónde la planificación de fuerza laboral diagnosticaba la mayor fotografía del negocio de la empresa, ya que se le veía como relacionándose con la función de personal, la cual en ese tiempo era todavía una función operacional del día a día.

En la década de 1980 se vio el impacto de barreras económicas que florecían. En el sector público esto dio paso a un mayor enfoque en la planificación estratégica en negocios, y a finales de los 80 , se percibió un tiempo de cambio radical en términos de disminución y reestructuración. La planificación de la fuerza laboral se orientó más a los negocios, identificando el suministro futuro y la necesidad de desarrollar habilidades a través de planificación en menor tiempo y el uso de una variedad más amplia de herramientas y técnicas. Esto requirió de administradores, líderes de grupo y profesionales de recursos humanos que trabajaron juntos para integrar la planificación de la fuerza laboral dentro de los procesos de planificación de la empresa.

En Puerto Rico son escasos los estudios realizados para determinar la fuerza laboral (Rivera Aponte, 2006; Ruiz Mercado 1986). 
Análisis histórico de LA PROFEsión de RECURSOS humanos en PueRto Rico...

Debemos señalar que las pocas investigaciones realizadas sobre la fuerza laboral en la isla se han realizado a nivel macro y no existen estudios en profundidad por áreas profesionales. El objetivo de este estudio es determinar la evolución, el desarrollo y la trayectoria de la fuerza laboral en las ocupaciones relacionadas con la disciplina de recursos humanos a partir del 1960 al 2000, por género; si existe o no una brecha salarial, y la cantidad de ofrecimientos académicos en el nivel de educación superior en Puerto Rico. La investigación surge dado que en Puerto Rico no existen estudios específicos en este campo o disciplina de la profesión de los recursos humanos. La nueva visión del desarrollo debe estar centrada en los recursos humanos. El desarrollo económico es equivalente al desarrollo de las competencias de la personas, de sus habilidades, capacidades, de sus recursos y motivaciones.

\section{REVISIÓN DE LITERATURA}

\section{Definición de RECURSOS HUMANOS Y COMPARACIÓN VIS A VIS GERENCIA DE PERSONAL}

A lo largo de las décadas, el concepto de recursos humanos ha sido continuamente confundido con el concepto del manejo de personal y del desarrollo de los recursos humanos (Rudman, 2010). El término de gerencia de los recursos humanos se ha desarrollado a partir de la gerencia del personal, a principios del siglo XX, y actualmente grandes compañías lo denominan bajo el término de gerencia del capital humano (Haslinda, 2009). Existen diferencias sustanciales entre la gerencia de personal y la gerencia de los recursos humanos. Según Sukumar (2011), ambos términos se distinguen en la siguiente tabla:

Tabla 1

Diferencias entre la gerencia de los recursos humanos y la gerencia del personal

\begin{tabular}{|c|c|c|}
\hline Puntos de diferencia & $\begin{array}{c}\text { Gerencia de los recursos } \\
\text { humanos }\end{array}$ & Gerencia de personal \\
\hline Función clave & $\begin{array}{c}\text { Mejorar la eficiencia } \\
\text { de la organización }\end{array}$ & $\begin{array}{c}\text { Leyes laborales y manejo de } \\
\text { pago de nóminas }\end{array}$ \\
\hline Enfoque & $\begin{array}{c}\text { Crear una cultura } \\
\text { dinámica }\end{array}$ & Administrar el personal \\
\hline
\end{tabular}


Rivera Aponte / Latortue Albino

\begin{tabular}{|c|c|c|}
\hline Puntos de diferencia & $\begin{array}{l}\text { Gerencia de los recursos } \\
\text { humanos }\end{array}$ & Gerencia de personal \\
\hline Diseño del trabajo & Trabajo en grupo & División de las labores \\
\hline Estandarización & Baja & Alta \\
\hline Pago & $\begin{array}{l}\text { Relacionado con el } \\
\text { rendimiento }\end{array}$ & $\begin{array}{c}\text { Relacionado con la evaluación } \\
\text { del trabajo }\end{array}$ \\
\hline Intervención & $\begin{array}{l}\text { Amplio margen de } \\
\text { estrategias }\end{array}$ & $\begin{array}{l}\text { Métodos en base a } \\
\text { procedimiento }\end{array}$ \\
\hline Rol de la gerencia & Transformación & Transaccional \\
\hline $\begin{array}{l}\text { Método de } \\
\text { comunicación }\end{array}$ & Directo & Indirecto \\
\hline $\begin{array}{l}\text { Habilidades claves } \\
\text { para la gerencia }\end{array}$ & Facilitar & Negociar \\
\hline Contratos gerenciales & Contratos individuales & $\begin{array}{l}\text { Contratos colectivos o de la } \\
\text { Unión }\end{array}$ \\
\hline Tarea gerencial & Nutrir & Monitorear \\
\hline Gerentes claves & $\begin{array}{l}\text { Negocios/líneas/ } \\
\text { gerentes generales }\end{array}$ & $\begin{array}{l}\text { Gerencia de Personal o } \\
\text { Gerencia de Relaciones } \\
\text { Industriales }\end{array}$ \\
\hline $\begin{array}{l}\text { Naturaleza de la } \\
\text { relación }\end{array}$ & Unitario & Pluralismo \\
\hline Acción gerencial & $\begin{array}{c}\text { Urgencia informal } \\
\text { para las necesidad del } \\
\text { negocio }\end{array}$ & Procedimientos \\
\hline Toma de decisiones & Rápida & Lenta \\
\hline Conflicto & Enfatizar & Institucionalizar \\
\hline Relación clave & Cliente & Labor \\
\hline
\end{tabular}

Fuente: Suganya Sukumar (2011).

Estas diferencias antes expuestas gestan las bases para la discusión y el análisis sobre el comportamiento y desarrollo de este campo de estudio. Los recursos humanos como profesión incluyen la atracción, el desarrollo, el sostenimiento del talento y de la energía de la fuerza trabajadora (Schermerhorn, 2008). La función de los recursos humanos es vital, ya que es un componente integral dentro de las estrategias de la gerencia para poder alcanzar las metas y el éxito organizacional. Mediante los recursos 
Análisis histórico de la profesión de reCursos humanos en Puerto Rico...

humanos, se manejan aspectos legales, adiestramiento, selección, sistemas de compensación, beneficios, relación obrero-patronal, planificación de los recursos humanos y todas las estrategias necesarias de la gerencia de los recursos humanos para implementar las estrategias organizacionales (Schermerhorn, 2008). Sin embargo, esta visión de las funciones que desempeñan los recursos humanos no siempre predominó en las organizaciones. Para entender dicho planteamiento procederemos a evaluar la evolución del manejo del personal en Europa, Estados Unidos y Puerto Rico, y la diferencia que existe hoy día entre los recursos humanos y el concepto de gerencia de personal que surge a partir de distintos eventos políticos, económicos y tecnológicos, y que más adelante se van a diferenciar y evolucionan en lo que conocemos como los recursos humanos.

\section{Evolución de los Recursos Humanos}

\section{ERA INDUSTRIAL}

Los principales eventos de los orígenes de la administración surgen en Egipto aproximadamente en 400 A.C.; generan el reconocimiento de la necesidad de planificar, organizar y controlar (Chiavenato, 1995; Khandwalla, 1977). Sin embargo, el surgimiento de la disciplina de los recursos humanos arranca del estudio del inicio y la evolución de la gerencia del personal, base fundamental para estudiar la evolución del campo de los recursos humanos. ¿Dónde nace la profesión de los recursos humanos? Según nos presentan Haines III, Brouillard y Cadieux (2010), la industrialización, los sindicatos, las uniones, las grandes compañías, así como las leyes relacionadas con el derecho al trabajo, dieron base al desarrollo de la profesión de los recursos humanos.

El origen de la gerencia de personal data de la era industrial (Rudman, 2010). La industrialización emerge en países europeos, específicamente en Inglaterra, durante el siglo XVIII. Este movimiento económico nace en dicho país, ya que poseía yacimientos de carbón (combustible utilizado en esa época) y de hierro, con los cuales se construyeron ferrocarriles y barcos (medios de transportación de los productos creados para transportar productos manufacturados de 
las industria). La Revolución Industrial significó una concentración de empleo en las industrias que, entre otros productos, trabajaban con el algodón. Esta revolución implicó un cambio en el estilo de vida y de trabajo de la fuerza laboral del país. Así, muchísimos trabajadores de distintos países europeos, provenientes de la industria agrícola, optaron por incorporarse a empleos propios del sector manufacturero (Rudman, 2010). Este cambio promovió el desarrollo de maquinarias y ocasionó implicaciones sociales, económicas ${ }^{1}$, políticas, demográficas, entre otras. Los empleados que trabajaban en dichas manufactureras eran percibidos como un costo para la compañía, cuyo enfoque consistía en producir a gran escala, al menor costo posible. Los empleados trabajaban por largos periodos, con un pago muy bajo.

Cómo nos explica Richard Rudman en su escrito Human Resource Management in New Zealand (2010), la mayoría de estos empleados, quienes incluían niños, trabajaban por largas horas, por un sueldo bajo y en condiciones que ofrecían poca protección contra temperaturas extremas, ruidos, desprovistos de seguridad en general. Otro aspecto importante que señala Rudman (2010) en su escrito, es que estos trabajadores de las industrias manufactureras repetían una y otra vez la misma operación dentro de la producción, a diferencia de la variedad de trabajos que posiblemente podían realizar dentro del sector agrícola. En 1802, el gobierno inglés pasó una ley para proteger a dichos jóvenes y niños, reduciendo sus horas laborables a 12 horas diarias, sin embargo, en la práctica dicha ley no fue respetada (Gordon, 2000).

En los años 1800, la fuerza trabajadora emprendió la reclamación de sus derechos, a pesar de que no fue hasta 1842, cuando en los Estados Unidos votaron por el derecho de organizarse colectivamente en uniones. Con la creación de uniones se ejercía presión al Gobierno y a raíz de las grandes manifestaciones en contra de las condiciones de trabajo y el bajo salario, de parte de las uniones y de la fuerza trabajadora, en 1833 el gobierno británico pasó un acta,

\footnotetext{
${ }^{1}$ En 1817, David Ricardo (1772-1823) publicó Principios de economía y tributación, texto centrado en el estudio del trabajo (enfocado como elemento de costo), el capital, el salario, la renta, la producción, los precios y el mercado. 
llamada Factory Act, como medida para mejorar las condiciones de salud y de seguridad en las cuales se trabajaba en las industrias. Algunas de las medidas que incluía dicha acta, eran: ningún niño menor de 9 años podía trabajar en las industrias; niños entre 9-13 no podían trabajar más de 12 horas al día; ningún niño podía trabajar en la noche y estos debían recibir 2 horas de educación diaria; las maquinarias peligrosas debían estar aseguradas. Esta acta dio base para futuras reformas. En cuanto a salario recibido, según datos presentados por Rudman (2010), tanto en Gran Bretaña, como en los Estados Unidos, los empleados recibían unos cupones de compra que podían ser utilizados solo dentro de la tienda de la compañía. Esto implicaba un posible abuso en los precios o una limitación en la ofertas de los productos que podían adquirir los empleados. Con el Truck Act de 1891 en Gran Bretaña, por primera vez se requería el pago de salario en efectivo.

A lo largo de los años subsiguientes, comienzan a surgir programas de bienestar, concentrados en mejorar las condiciones del trabajo dentro de las industrias. Sin embargo, antes de que se fomentaran y se incluyeran cambios en el bienestar de estos trabajadores, a nivel intelectual, grandes científicos y pensadores estudiaron y analizaron el impacto que tenían las condiciones de trabajo y la forma de operar en las distintas industrias, en sus empleados. Podemos destacar a nivel teórico, tres movimientos claves que dan inicio al marco teórico de los recursos humanos. El primero es el marco científico gerencial que se puede resumir en varios principios destacados por Frederick Winslow Taylor, mejor conocido como el padre del movimiento científico de gerencia. Para 1920, el ingeniero americano Frederick Winslow Taylor introdujo junto a colegas, estudios científicos de gerencia dirigidos a los líderes de las empresas, con el propósito de que científicamente se comprobaran técnicas que mejoraran la productividad y la eficiencia de una compañía. Entre los principios que Frederick Winslow desarrolló, Gerald Ngugi Kimani los segregó en los siguientes: primero, la utilización de un tiempo determinado para medir la productividad. Segundo, la paga debía ser proporcional al resultado trabajado; en otras palabras, a mayor trabajo realizado, mayor la recompensa salarial. Tercero, la división de las labores, esto es, se separaban la 
tarea administrativa de planificación, la facilitación de las tareas y la de motivar, de la del trabajo de los empleados enfocados en llevar a cabo las tareas que aumenten la productividad y eficiencia de las organizaciones. Cuarto, y último principio, es el de especialización. Bajo este principio, se esperaba que los que poseían la autoridad tuvieran el dominio teórico y las habilidades sobre lo que estaban supervisando (Rudman 2010; Chiavenato, 1995; Taylor, 1911).

Otro estudioso destacado en el movimiento fue Robert Owen, inglés quien a la edad de los 18 años fue fundador de su propia empresa, defensor de la educación, de la salud y de las condiciones de trabajo de los niños y jóvenes que trabajaban en las distintas industrias. En su propias plantaciones de algodón, Owen no permitió a niños trabajar en la recolecta, y a su vez mejoró las instalaciones sanitarias y proveyó educación a los niños y a los trabajadores. Para esa misma década, títulos como labor manager y employment manager fueron utilizados en distintas industrias para resolver problemas como el ausentismo, los reclutamientos, entre otros aspectos del día a día en las industrias.

\section{Comienzo de los Recursos humanos}

Para la década de 1930, grandes corporaciones comenzaron a valorar medidas que luego formarían parte de lo que conocemos hoy día como recursos humanos. Dichas corporaciones, según nos presentan Daniels, Argree y Davis (2008), reconocieron el valor de mejorar los beneficios de los empleados para poder reclutarlos, retenerlos y motivarlos a su vez. A mayor beneficio y motivación, mayor era la posibilidad de un incremento en la productividad de los trabajadores. Sin embargo, otras industrias como la de textiles, no vieron la necesidad de estos beneficios, ya que para ellos reclutar no presentaba mayor dificultad. Con el crecimiento de grandes compañías, entre los años 60, las corporaciones aprovecharon el avance tecnológico para cumplir su necesidad de centralizar los datos personales y mantener un récord actualizado, lo cual originó el nacimiento del human resources software. Otro factor clave para el surgimiento de los recursos humanos es lo que se conoce hoy día como el movimiento de la conducta científica. 
Análisis histórico de la profesión de ReCursos humanos en Puerto Rico...

No obstante, durante estos periodos no se estudiaba la planificación de la fuerza laboral.

\section{Recursos humanos en Puerto Rico}

Según Santana (1996), la historia indica que las primeras inquietudes y esfuerzos comienzan cuando los norteamericanos llegan a Puerto Rico en el 1898. Apenas hacía 15 años que en el continente americano, a nivel federal, se había aprobado su primera ley de servicio civil de mérito (Ley Pendleton, de 1883), después de haber transcurrido un siglo de patronazgo, de tragedias y de corrupción. Inmediatamente que los nuevos gobernantes tomaron el control de Puerto Rico e instauraron un gobierno militar, empezaron a indagar sobre las impresiones y la capacidad de los puertorriqueños para administrar los asuntos de gobierno bajo el control y la tutela del nuevo imperio, especialmente en el área de servicio civil. Aprovechando la efervescencia habida en los Estados Unidos con la aprobación de la Ley Pendleton, era obvio que los primeros gobernantes americanos se interesaran en informar a Washington sobre la importancia y deseabilidad de aprobar para Puerto Rico "una ley general de servicio civil como la única base segura y satisfactoria para edificar desde los primeros momentos la formación de un gobierno local" (Santana, 1993).

De este modo, bajo el contagio de la consigna de asegurar un gobierno "satisfactorio para el nuevo territorio", se presentó en el 1905 un proyecto de servicio civil de mérito, el cual no prosperó pero, no obstante, sentó las bases para que inmediatamente en el 1907 se aprobara con la misma finalidad, la primera ley de administración de personal. Santana (1996; Santana, 1993) indica que durante el transcurso del presente siglo, en Puerto Rico se han realizado cuatro intentos legislativos para dotar a nuestro servicio civil de un sistema de mérito. En 1907, 1931, 1947 y 1975 se aprobaron con renovado elogio y devoción sendas leyes, dirigidas cada una de ellas a ampliar y enriquecer los postulados y las instituciones que la experiencia y el conocimiento crecientes, supuestamente, han venido abonando.

En los años 1907, 1931 y 1947 se aprobaron distintas leyes para tratar de implementar un sistema de servicio civil en Puerto Rico 
y adoptar el principio de mérito como norma fundamental de la administración de personal. En esencia, los diversos esfuerzos fracasaron por razones similares: la permanencia de discrímenes políticos, fallas de instrumentación, violaciones a las normas y procedimientos, e inadecuacidad del sistema de mérito para proteger a la mayoría de los empleados públicos (Ruíz Acevedo, 1996).

El 14 de octubre de 1975 se aprobó la Ley Número 5 (conocida como la Ley de Personal del Servicio Público de Puerto Rico), con el objetivo principal de reafirmar y extender el principio de mérito a todos los sectores del empleo público bajo un solo sistema de personal. Este se definió como:

...el principio que regirá todo el servicio público, de modo que sean los más aptos que sirvan al Gobierno y que todo empleado sea seleccionado, adiestrado, ascendido y retenido en su empleo en consideración al mérito y a la capacidad, sin discrimen por razones de raza, color, nacimiento, edad, origen o condición social, ni por ideas políticas o religiosas. (Santana, 1993, p. 55)

A tales fines se identificaron cinco áreas esenciales relacionadas con el principio de mérito: clasificación de puestos, reclutamiento y selección, ascenso, traslado y descensos, adiestramiento y retención (Santana, 1993). El sistema de mérito creado para instrumentar y darle vigilancia a dicho principio incluye y clasifica las agencias del Gobierno en tres grupos o componentes:

- Las agencias comprendidas en la Administración Central, las cuales serán reglamentadas, administradas y coordinadas por la Oficina Central de Personal (OCAP), sobre las cuales se delegarán funciones operacionales cuando sea conveniente.

- Administradores individuales que tienen la responsabilidad de administrar directamente todo lo relativo a su personal conforme con el reglamento que adopten en armonía con la Ley de personal y el Reglamento de personal.

- Áreas esenciales al principio del mérito y agencias excluidas de las disposiciones de la Ley de Personal: corporaciones públicas y la Universidad de Puerto Rico (Santana, 1993). 
A mediados de la década de los 1940, Puerto Rico pasó por unos cambios políticos en la economía, con la llegada de la gobernación de Rexford G. Tugwell, último estadounidense en ocupar este puesto. Estos cambios políticos causan que el desarrollo económico de la isla se concentre en programas estratégicos orientados hacia adentro, como los describe Irizarry Mora en su texto Economía de Puerto Rico (2011). Dichos programas se enfocaron en estrategias que favorecieron la creación de la industria manufacturera en Puerto Rico, dirigida por 4 grandes manufactureras que requerían un alto nivel de mano de obra. A este periodo histórico se le atribuyó el nombre del periodo de industrias livianas, entre los años 1948-1965. En 1947 se firmó la Ley de Incentivos Industriales. En 1948 se inició la estrategia de industrialización por invitación que, como nos expone Irizarry Mora en su texto, bajo la dirección de la Administración de Fomento Económico, promovió a Puerto Rico como el destino para las inversiones de manufactureras estadounidenses. De este modo se dio inicio al programa de desarrollo industrial, llamado Operación Manos a la Obra. Estas estrategias son vitales para nuestro estudio de los recursos humanos, ya que el establecimiento de estas fábricas manufactureras requería una mano de obra intensiva.

Según Ruíz Mercado (1990) en su escrito Nueva estrategia de desarrollo, los trabajadores eran vistos como parte de los demás insumos utilizados para producir bienes y servicios. En pocas palabras, los trabajadores representaban un costo necesario para poder lograr los objetivos de producción establecidos por la compañía. El trabajo en las manufactureras se medía por las unidades producidas.

\section{Recursos humanos hoy día}

Evaluando los cambios que experimentan las organizaciones hoy día, debido a los rápidos cambios tecnológicos, ambientales, el incremento de la competencia y los cambios en la economía global, es evidente que las distintas funciones dentro de la organizaciones han tenido que evolucionar y desarrollarse utilizando nuevas estrategias que le añadan valor y permitan a la organización ganar una ventaja competitiva a largo plazo sobre las demás. Atrás quedó la sola responsabilidad de recursos humanos, enfocada solo en 
su personal y en su reclutamiento, selección, adiestramiento, motivación y/o el desarrollo del talento dentro de una organización. Hoy día recursos humanos juega un papel clave en las estrategias por seguir para lograr el crecimiento y desarrollo sustentable de las organizaciones. Según nos presenta CIPD (Charted Institute of Personnel and Development), la organización compuesta de profesionales desarrollados y de recursos humanos más grande en Europa, los recursos humanos han pasado de ayudar a los gerentes a manejar bien su personal, a proveer sustentabilidad y eficiencia a la organización en términos de su capacidad y de su desempeño organizacional. Nos preguntamos ¿cuál es la fuerza laboral en Puerto Rico en la ocupación de los recursos humanos?

\section{Metodología}

Las fuentes principales para realizar esta investigación se obtuvieron de los archivos del Consejo de Educación de Puerto Rico. En estas fuentes se encuentran los programas académicos a nivel subgraduado y graduado de todas las instituciones académicas a nivel de educación superior que están autorizados por el Consejo de Educación Superior en la isla. Estos datos se clasificaron por niveles académicos desde grados asociados, bachilleratos, maestrías, doctorados y certificados a nivel universitario para la disciplina del campo de recursos humanos.

La segunda fuente principal fueron los documentos publicados por el Departamento de Comercio de los Estados Unidos de América, el Negociado del Censo Federal, el Censo de Población de Puerto Rico durante las décadas de 1960, 1970, 1980, 1990 y 2000. También se solicitó información adicional a la Oficina de Censo Federal, la Oficina del Censo de Puerto Rico, la Junta de Planificación de Puerto Rico y la Universidad de Puerto Rico. De esas fuentes se recopilaron los datos concernientes a la clasificación ocupacional detallada del grupo trabajador civil diestro y de las personas empleadas según su sexo y salario, para determinar la fuerza laboral de la profesión de los recursos humanos.

Parte de los datos del Censo de 2000 se obtuvieron a través de llamadas telefónicas y de comunicación electrónica con el Negociado 
Análisis histórico de la profesión de recursos humanos en Puerto Rico...

del Censo Federal, debido a que el banco de datos censales disponible en Puerto Rico estaba sujeto a ciertas restricciones. Estas últimas se relacionan específicamente con los datos informados de las ocupaciones e industrias. La información de clasificación industrial y ocupacional por género se recibió a mediados del mes de marzo del 2005 a través de una autorización del gobierno federal de los Estados Unidos, respondiendo a una petición especial de Rivera Aponte (2006) para utilizar los archivos de Advance Query Systems (AQS). Estos archivos fueron trasladados al programa Excel de manera desagregada. Por su amplitud, se utilizó un sistema computarizado especial para poder limpiarlos (AQS enviados por BLS de Estados Unidos). La información fue clasificada en tres vertientes: ocupación por género, ocupación por industria, y ocupaciones que agrega el campo de la profesión de los recursos humanos. Para el análisis de los datos se utilizó el Modelo de Matriz de Insumo-Producto de Leontief (Leontief, 1953).

Se clasificaron todas las ocupaciones utilizando el Sistema de clasificación ocupacional de los Estados Unidos (1960, 1970, 1980, 1990 y 2000), usando diferentes niveles de agregación: grupo ocupacional principal, subgrupos ocupacionales y detalle de cada una de las ocupaciones. La magnitud de las ocupaciones registradas en Puerto Rico a través de las décadas, los cambios en los nombres de las clasificaciones y de los códigos que establecen los estándares de clasificaciones ocupacionales (SOC), requirieron uniformar la base de datos.

A continuación se clasificaron cada una de las ocupaciones de manera detallada a través de las décadas. La cantidad de estas ocupaciones fluctuó entre 500 a 650. Después de identificar los códigos y definiciones establecidas en el manual de clasificación ocupacional, se agregaron las ocupaciones dividiéndolas en diferentes categorías, lo que resultó en una agregación de 113 subgrupos ocupacionales. Estos subgrupos ocupacionales a su vez se reagruparon en 13 grupos ocupacionales principales: las ocupaciones ejecutivas, administrativas y gerenciales; las profesionales; las técnicas y de apoyo técnico; los vendedores; las de apoyo administrativo, oficinista, secretarias y otros; las de servicios; las de agricultura, silvicultura y pesca; reparadores y mecánicos; trabajadores diestros en 
la construcción; las ocupaciones en trabajos de precisión; los operarios de transportación y ocupaciones relacionadas con el movimiento de materiales y los trabajadores de la construcción y otros obreros. Una vez completada la recopilación y clasificación de los datos, se hizo el análisis de los cambios en los empleos ocupados en el campo de recursos humanos para Puerto Rico, por género y salario. Además se determinó si existe o no la brecha de salario por género en las ocupaciones relacionadas con el campo de recursos humanos en Puerto Rico, para la década del 2000.

\section{Discusión Y ANÁLISIS}

\section{EDUCACIÓN Y RECURSOS HUMANOS}

Uno de los objetivos del estudio es determinar la cantidad de programas académicos en el nivel de educación superior en la disciplina académica de los recursos humanos en Puerto Rico. La misma será presentada por nivel académico e institución universitaria. Dicha información se obtuvo de la base de datos del Consejo de Educación Superior de Puerto Rico. Debemos señalar que los primeros cursos en esta disciplina en Puerto Rico se ofrecieron a mediados de 1940 en la Facultad de Administración Comercial y luego en la Facultad de Ciencias Sociales de la Universidad de Puerto Rico en el Recinto de Río Piedras. El primer curso ofrecido en la disciplina de recursos humanos fue economía laboral en el Departamento de Economía de la Universidad de Puerto Rico en Río Piedras después de 1947-48². Se diseñó y se creó en 1945 a nivel subgraduado la concentración de Administración Pública. Luego en 1947 se estableció la Escuela Graduada de Administración Pública; ${ }^{3}$ años más tarde se estableció el

\footnotetext{
${ }^{2}$ Según señalan Roca, Gaztambide y Morales (2003), una revisión curricular en 1947-48 reafirmó las concentraciones para cursarse después del segundo año. En ese año académico, el Departamento de Economía, importado como tal de la Facultad de Administración Comercial, se transformó en el Programa de Economía.

${ }^{3}$ Ley Núm. 26, 21 de noviembre de 1941 (18 LPRA 664). Véase también Leonardo Santana Rabell, Apuntes históricos sobre planes de estudios y cambios curriculares de la Escuela Graduada de Administración Pública: 1945-1996, Revista de Administración Pública, 28(1) (diciembre 1966), pp. 57-93; Charles T. 
Análisis histórico de la profesión de recursos humanos en Puerto Rico...

Programa de Administración de Personal en 1966; y luego, la Escuela de Comercio de la Universidad de Puerto Rico en el Recinto de Río Piedras, en 1977. En las Tablas 2, 3 y 4, se desglosa la cantidad de programas académicos relacionados con recursos humanos, por niveles académicos, por cientos y proporciones. Según la información obtenida de la base de datos del Consejo de Educación Superior en Puerto Rico para el 2010, en Puerto Rico existen unos 53 programas académicos entre diez instituciones universitarias. El Sistema de la Universidad Interamericana de Puerto Rico ofrece unos 17 programas académicos a nivel graduado y subgraduado. Esto representa el 32.1 por ciento de los ofrecimientos académicos en esta disciplina. La segunda institución académica con mayor ofrecimiento en la disciplina de recursos humanos es el Sistema Ana G. Méndez, con un 16.9 por ciento (nueve programas académicos a nivel graduado y subgraduado). Estas dos instituciones antes mencionadas ofrecen un total de 26 programas académicos (49.1 por ciento) del campo de los recursos humanos. El Sistema de la Universidad de Puerto Rico solamente ofrece un 9.0 por ciento (unos cinco programas académicos) de total de ofrecimientos en esta área de estudios en recursos humanos.

La Tabla 3 indica que del total de 53 programas académicos en recursos humanos, un 58.5 por ciento de ellos (31 programas) pertenecen al nivel graduados (29 a nivel de maestría y dos a nivel doctoral) y un 41.5 por ciento de los mismos corresponden al nivel subgraduados (17 a nivel de bachillerato y cinco grados asociados). El 86.8 por ciento de los programas se concentra en el nivel de bachillerato y maestría en Puerto Rico. Las instituciones de educación privada ofrecen el 81.0 por ciento de ellos (48 programas académicos), se concentran en 27 maestrías y 14 bachilleratos. Estos datos antes expuestos evidencian que en la isla se promueve una continua proliferación de creación, diseño, modificaciones y

Goodsell, Administración de una revolución: la reforma del poder ejecutivo de Puerto Rico bajo el Gobernador Tugwell (1941-1946), Trad. de Pedro Salazar, San Juan: Editorial Universitaria, 1967, pp. 128-129; Ángel Quintero Rivera, La ideología populista y la institucionalización de las ciencias sociales. En Silvia Álvarez Curbelo y María Elena Rodríguez Castro (Eds), Del nacionalimo al populismo en Puerto Rico. Río Piedras: Ediciones Huracán. pp: 107-145. 
Cantidad de programas académicos relacionados con recursos humanos, según niveles académicos en instituciones de educación superior en Puerto Rico, para 2011

\begin{tabular}{|c|c|c|c|c|c|}
\hline \multirow[b]{2}{*}{ Nombre de la Institución } & \multicolumn{2}{|c|}{ Subgraduado } & \multicolumn{2}{|c|}{ Graduados } & \multirow{2}{*}{$\begin{array}{c}\text { Total de } \\
\text { Programas } \\
\text { Académicos }\end{array}$} \\
\hline & $\begin{array}{c}\text { Grados } \\
\text { Asociados }\end{array}$ & Bachilleratos & Maestrías & Doctorados & \\
\hline American University of Puerto Rico & 2 & 2 & 0 & 0 & 4 \\
\hline Bayamón Central University & 0 & 1 & 0 & 0 & 1 \\
\hline Caribbean University & 3 & 0 & 4 & 0 & 7 \\
\hline Columbia Centro Universitario & 0 & 0 & 1 & 0 & 1 \\
\hline Sistema de la Pontificia Universidad Católica & 0 & 1 & 4 & 0 & 5 \\
\hline Sistema Universidad de Puerto Rico & 0 & 3 & 2 & 0 & 5 \\
\hline Sistema Ana G. Méndez & $\mathbf{0}$ & 1 & 8 & 0 & 9 \\
\hline Sistema de la Universidad Interamericana & $\mathbf{0}$ & 9 & 6 & 2 & 17 \\
\hline Universidad del Sagrado Corazón & 0 & 0 & 1 & 0 & 1 \\
\hline University of Phoenix & 0 & 0 & 3 & 0 & 3 \\
\hline Total & 5 & 17 & 29 & 2 & 53 \\
\hline
\end{tabular}

Fuente: Oficina de Estadísticas, Consejo de Educación Superior de Puerto Rico. 
Tabla 3

Distribución porcentual de programas académicos relacionados con recursos humanos según niveles académicos en instituciones de educación superior en Puerto Rico, para 2011

\begin{tabular}{|c|c|c|c|c|c|}
\hline \multirow[b]{2}{*}{ Nombre de la Institución } & \multicolumn{2}{|c|}{ Subgraduado } & \multicolumn{2}{|c|}{ Graduados } & \multirow{2}{*}{$\begin{array}{c}\text { Total de } \\
\text { Programas } \\
\text { Académicos }\end{array}$} \\
\hline & $\begin{array}{c}\text { Grados } \\
\text { Asociados }\end{array}$ & Bachilleratos & Maestrias & Doctorados & \\
\hline American University of Puerto Rico & $50.0 \%$ & $50.0 \%$ & $0.0 \%$ & $0.0 \%$ & $100.0 \%$ \\
\hline Bayamón Central University & $0.0 \%$ & $100.0 \%$ & $0.0 \%$ & $0.0 \%$ & $100.0 \%$ \\
\hline Cambridge College & $42.9 \%$ & $0.0 \%$ & $57.1 \%$ & $0.0 \%$ & $100.0 \%$ \\
\hline Caribbean University & $42.9 \%$ & $0.0 \%$ & $57.1 \%$ & $0.0 \%$ & $100.0 \%$ \\
\hline Sistema de la Pontificia Universidad Católica & $0.0 \%$ & $20.0 \%$ & $80.0 \%$ & $0.0 \%$ & $100.0 \%$ \\
\hline Sistema Universidad de Puerto Rico & $0.0 \%$ & $60.0 \%$ & $40.0 \%$ & $0.0 \%$ & $100.0 \%$ \\
\hline Sistema Ana G. Méndez & $0.0 \%$ & $11.1 \%$ & $88.9 \%$ & $0.0 \%$ & $100.0 \%$ \\
\hline Sistema de la Universidad Interamericana & $0.0 \%$ & $52.9 \%$ & $35.3 \%$ & $11.8 \%$ & $100.0 \%$ \\
\hline Universidad del Sagrado Corazón & $0.0 \%$ & $0.0 \%$ & $100.0 \%$ & $0.0 \%$ & $100.0 \%$ \\
\hline \multirow[t]{2}{*}{ University of Phoenix } & $0.0 \%$ & $0.0 \%$ & $100.0 \%$ & $0.0 \%$ & $100.0 \%$ \\
\hline & $9.4 \%$ & $32.1 \%$ & $54.7 \%$ & $3.8 \%$ & $1000.0 \%$ \\
\hline
\end{tabular}

Fuente: Oficina de Estadísticas, Consejo de Educación Superior de Puerto Rico. 
revisiones académicas en todos los niveles y en todas las instituciones a nivel de educación superior. Sin embargo, la falta de planificación de los recursos humanos promueve el continuo desarrollo y la generación de programas académicos sin integrar la oferta y la demanda del mercado laboral en Puerto Rico (Rivera Aponte, 2006).

La Tabla 4, muestra la proporción de los programas académicos en el campo de los recursos humanos, lo que deberá ser estudiado cuidadosamente por las entidades reguladoras de educación superior en Puerto Rico. A nivel subgraduado, en grados asociados se ofrecen cinco programas académicos entre la American University (una proporción de $40.0 \%$ ) y Colombia University (una proporción de $60.0 \%$ ). También debemos indicar que el Sistema de la Universidad Interamericana a nivel subgraduado ofrece bachillerato en recursos humanos en nueve de sus diez unidades académicas (una proporción de 52.4\%) mientras que el Sistema de Educación Pública de la Universidad de Puerto Rico ofrece tres programas, en tres de sus once unidades académicas (una proporción de 17.6\%). En términos proporcionales, estos dos sistemas universitarios ofrecen un total de 13 de 17 programas académicos a nivel de bachillerato (una proporción de $76.4 \%$ ).

También debemos señalar que en Puerto Rico existen 29 maestrías en el campo de los recursos humanos, de las cuales 27 (una proporción $93.1 \%$ ) se ofrecen en las instituciones privadas de educación superior en la isla. A nivel doctoral, solamente las universidades privadas ofrecen estudios doctorales en áreas relacionadas en recursos humanos en administración de empresas.

\section{Tendencias Generales en el EMPLEO EN LA ocupación DE LOS RECURSOS HUMANOS Y GÉNERO}

Las bases de datos referentes para realizar la comparación sobre empleo en Puerto Rico han evidenciado una gran discrepancia (Rivera Aponte, 2006). Por ejemplo, para el año 2000 los datos de empleo publicados por el Departamento del Trabajo totalizaron $1,159,000$ mientras que en los datos censales el empleo totalizaba $1,569,934$. Aunque los datos del censo se toman en periodos diferentes del año (1999) y los datos del Departamento del Trabajo son para 
Tabla 4

Proporción de programas académicos relacionados con recursos humanos según niveles académicos en instituciones de educación superior en Puerto Rico, para 2011

\begin{tabular}{|c|c|c|c|c|c|}
\hline \multirow[b]{2}{*}{ Nombre de la Institución } & \multicolumn{2}{|c|}{ Subgraduado } & \multicolumn{2}{|c|}{ Graduados } & \multirow{2}{*}{$\begin{array}{c}\text { Total de } \\
\text { Programas } \\
\text { Académicos }\end{array}$} \\
\hline & Grados Asociados & Bachilleratos & Maestrias & Doctorados & \\
\hline American University of Puerto Rico & 40.00 & 11.76 & 0.00 & 0.00 & 7.55 \\
\hline Bayamón Central University & 0.00 & 5.88 & 0.00 & 0.00 & 1.89 \\
\hline Caribbean University & 60.00 & 0.00 & 13.79 & 0.00 & 13.21 \\
\hline Sistema de la Pontificia Universidad Católica & 0.00 & 5.88 & 13.79 & 0.00 & 9.43 \\
\hline Sistema Universidad de Puerto Rico & 0.00 & 17.65 & 6.90 & 0.00 & 9.43 \\
\hline Sistema Ana G. Méndez & 0.00 & 5.88 & 27.59 & 0.00 & 16.98 \\
\hline Sistema de la Universidad Interamericana & 0.00 & 52.94 & 20.69 & 100.00 & 32.08 \\
\hline Universidad de Sagrado Corazón & 0.00 & 0.00 & 3.45 & 0.00 & 1.89 \\
\hline \multirow[t]{2}{*}{ University of Phoenix } & 0.00 & 0.00 & 10.34 & 0.00 & 5.66 \\
\hline & 100.00 & 100.00 & 100.00 & 100.00 & 100.00 \\
\hline
\end{tabular}

Fuente: Consejo de Educación Superior de Puerto Rico. 
años fiscales, la diferencia para ese año es tan grande que merece que las agencias gubernamentales encargadas de estos estimados ofrezcan una explicación. Para propósitos de investigación utilizamos la base de datos censales. Una de las razones principales es por el nivel de desagregación de las ocupaciones para la isla. Esta gestión se comenzó en el 2006. Para el nivel de integración y uniformidad (de más de 800 ocupaciones) de la bases de datos se utilizó el Diccionario de Ocupación del Trabajo, generado por el gobierno federal de los Estados Unidos de América (Rivera Aponte, 2006).

La Tabla 5 presenta la cantidad total de empleos generados en Puerto Rico en términos absolutos desde el 1960 al 2000. En la isla se generaron un total de 508,838 empleos (una tasa de crecimiento de $4.0 \%)$. Al examinar los datos encontramos que en las ocupaciones de los recursos humanos se evidenció un crecimiento hasta la década del 1990, el cual generó una cantidad de 10,109 empleos (una tasa de crecimiento de $10.8 \%$ ). Para el 2000, esta ocupación mostró una disminución de 2,527 empleos.

En la Tabla 6 se muestra la proporción de empleos en la ocupación de recursos humanos por décadas frente a la totalidad de empleos en Puerto Rico. El campo de los recursos humanos representa para la década del 2000 una proporción de 1.69\% (unos 26,516 empleos). El aumento de la fuerza laboral en la ocupación de los recursos humanos se debe en gran medida a los modelos de desarrollo económico generados en Puerto Rico durante los periodos de 1950 a 1990. Para finales de la década de 1950 al 1960, se habían promovido más de 500 plantas (filiales) y se habían asistido a más de 80 para una inversión combinada de más de $\$ 500$ millones y una creación de 40,000 nuevos empleos. Para finales de 1960 al 1970, ya existían unas 1,400 plantas las cuales eran responsables de dos terceras partes del empleo en la manufactura ${ }^{4}$. Gran parte de las empresas que operaban durante los primeros años del programa eran industrias livianas de alto contenido de mano de obra. Lo antes expuesto demuestra que la isla necesitaba personal diestro especializado en la ocupación de administración de personal con el objetivo de contribuir a maximizar los recursos de la empresa.

\footnotetext{
4 The Chase Manhattan Bank, Industry in Puerto Rico, julio 1967, página 10.
} 
Tabla 5

Comparación del empleo total y el empleo en la ocupación de recursos humanos, Puerto Rico, años censales 1960-2000

\begin{tabular}{|c|c|c|c|c|c|}
\hline & 1960 & 1970 & 1980 & 1990 & 2000 \\
\hline \multicolumn{6}{|l|}{ Valor Absoluto } \\
\hline Empleos Totales en Puerto Rico & 583,743 & 667,906 & 813,915 & $1,061,096$ & $1,569,934$ \\
\hline \multirow[t]{2}{*}{ Empleos Totales en Ocupación de Mercadeo } & 329 & 2,039 & 5,676 & 15,785 & 13,258 \\
\hline & $1960-70$ & $1970-80$ & $1980-90$ & $1990-2000$ & \\
\hline Empleos Totales en Puerto Rico & 84,163 & 146,009 & 247,181 & 508,838 & \\
\hline Empleos Totales en Ocupación de Mercadeo & 1,710 & 3,637 & 10,109 & $(2,527)$ & \\
\hline \multicolumn{6}{|l|}{ Tasas de Crecimiento } \\
\hline Empleos Totales en Puerto Rico & $1.4 \%$ & $2.0 \%$ & $2.7 \%$ & $4.0 \%$ & \\
\hline Empleos Totales en Ocupación de Mercadeo & $20.0 \%$ & $10.8 \%$ & $10.8 \%$ & $-1.7 \%$ & \\
\hline
\end{tabular}

Fuente: Departamento de Comercio Federal, Negociado del Censo, Censo de Población; 1960 al 2000 
Tabla 6

Demanda ocupacional total en recursos humanos en Puerto Rico.

Datos de los censos de 1960, 1979, 1980, 1990 y 2000

\begin{tabular}{|c|c|c|c|c|c|}
\hline \multirow[b]{2}{*}{ Grupo Ocupacional } & \multicolumn{5}{|c|}{ Empleo (Demanda) } \\
\hline & 1960 & 1970 & 1980 & 1990 & 2000 \\
\hline Demanda Total para Puerto Rico & 583,743 & 667,906 & 813,915 & $1,061,096$ & $1,569,934$ \\
\hline Demanda Total en Recursos Humanos para Puerto Rico & 329 & 2,039 & 5,676 & 15,785 & 13,258 \\
\hline $\begin{array}{l}\text { Proporción de Personas empleadas en } \\
\text { Recursos Humanos }\end{array}$ & $0.06 \%$ & $0.31 \%$ & $0.70 \%$ & $1.49 \%$ & $0.84 \%$ \\
\hline
\end{tabular}

Fuente: Department of Commerce. Census Bureau. Census of Population, Detailed Characteristics. 1960, 1970, 1980,1990 y 2000. 
Análisis histórico de la profesión de ReCursos humanos en Puerto Rico...

Los datos de empleo para la ocupación de recursos humanos por género, según publicados en la sección de estadísticas demográficas y económicas de los censos de población, se muestran en la Tabla 7.

Tabla 7

Empleo en la ocupación de recursos humanos según género, Puerto Rico, años censales 1960-2000

\begin{tabular}{|c|c|c|c|c|c|}
\hline \multirow[b]{2}{*}{ Valor Absoluto } & \multirow[t]{2}{*}{1960} & \multirow[t]{2}{*}{1970} & \multirow[t]{2}{*}{1980} & \multirow[t]{2}{*}{1990} & \multirow[t]{2}{*}{2000} \\
\hline & & & & & \\
\hline \multirow{3}{*}{$\begin{array}{l}\text { Empleos Totales en } \\
\text { Ocupación de Mercadeo } \\
\text { Masculino } \\
\text { Femenino }\end{array}$} & 329 & 2,039 & 5,676 & 15,785 & 13,258 \\
\hline & 246 & 1,326 & 3,383 & 9,583 & 5,788 \\
\hline & 83 & 713 & 2,293 & 6,202 & 7,470 \\
\hline \multirow{3}{*}{ Empleos Totales } & $1960-70$ & $1970-80$ & $1980-90$ & $1990-2000$ & \\
\hline & 1,710 & 3,637 & 10,109 & $(2,527)$ & \\
\hline & 1,080 & 2,057 & 6,200 & $(3,795)$ & \\
\hline Femenino & 630 & 1,580 & 3,909 & 1,268 & \\
\hline \multicolumn{6}{|l|}{ Tasas de Crecimiento } \\
\hline Total & $20.0 \%$ & $10.8 \%$ & $10.8 \%$ & $-1.7 \%$ & \\
\hline Masculino & $18.3 \%$ & $9.8 \%$ & $11.0 \%$ & $-4.9 \%$ & \\
\hline Femenino & $24.0 \%$ & $12.4 \%$ & $10.5 \%$ & $1.9 \%$ & \\
\hline
\end{tabular}

Fuente: Departamento de Comercio Federal, Negociado del Censo, Censo de Población; 1960 al 2000.

Los resultados del censo de la década de 1960 para la ocupación de recursos humanos, mostraron que en Puerto Rico existía un total de 329 personas empleadas de las cuales $83.0 \%$ eran de género masculino y $17.0 \%$ del género femenino. Una década más tarde, el empleo total aumentó a 2,039, un incremento absoluto de 1,710 y una tasa de crecimiento anual promedio de $20.0 \%$. Del total del aumento en el empleo desde 1960 a 1970, unos 1,080 correspondieron al 
sexo masculino y un incremento de 630 fue el aumento absoluto en el empleo femenino. Sin embargo, la tasa de crecimiento anual de un $24.0 \%$ para el género femenino, fue más alta que el género masculino por $18.3 \%$. Este patrón de comportamiento en el empleo por género ha continuado hasta el presente. O sea, más crecimiento absoluto y mayor tasa de crecimiento en el empleo femenino que en el masculino.

No obstante, debemos señalar que entre las décadas del 1990 al 2000 la cantidad total de empleos generados para el campo de los recursos humanos reflejó una disminución de 2,527 empleos. Esto representa una tasa de crecimiento anual de -1.7. Al realizar el análisis por la variable de género, encontramos que la disminución sustancial fue en el género masculino: unos 3,795 empleos (una tasa de crecimiento de $-4.9 \%$ ). Simultáneamente, para la misma década, el género femenino continuó mostrando un crecimiento de 1,268 empleos (un aumento en la tasa de crecimiento 1.9\%).

Según los datos del censo del 2000, en Puerto Rico este primer grupo ocupacional principal representa unos 124,470 empleos (Tabla 8). Este total de empleo significa una proporción de $7.9 \%$. De cada 100 empleos, siete fueron generados para este grupo ocupacional principal. Al profundizar más detalladamente en este grupo ocupacional principal de ejecutivos, administrativos y gerentes, podemos mencionar que una proporción de $10.7 \%$ representa las ocupaciones relacionadas con el campo de los recursos humanos $(13,258 / 124,470$ empleos es igual al 10.7\%). Estas ocupaciones son las siguientes: (a) administradores de personal y relaciones laborales, (b) especialistas en personal, adiestramiento, y relaciones laborales y (c) analistas gerenciales. 
Tabla 8

Empleo en la ocupación de recursos humanos por grupo ocupacional principal,

\begin{tabular}{|c|c|c|c|c|c|c|}
\hline \multicolumn{7}{|c|}{ Puerto Rico, años } \\
\hline & & 1960 & 1970 & 1980 & 1990 & 2000 \\
\hline & \multicolumn{6}{|l|}{ Valor Absoluto } \\
\hline & Empleos Totales en Puerto Rico & 583,743 & 667,906 & 813,915 & $1,061,096$ & $1,569,934$ \\
\hline & $\begin{array}{l}\text { Total Ocupaciones Ejecutivas, Administrativas y } \\
\text { Gerenciales para Puerto Rico }\end{array}$ & 32,250 & 44,992 & 63,717 & 99,143 & 124,470 \\
\hline & $\begin{array}{l}\text { Total de Ocupaciones Ejecutivas, Administrativas } \\
\text { y Gerenciales en Recursos Humanos para Puerto Rico }\end{array}$ & 329 & 2,039 & 5,676 & 15,785 & 13,258 \\
\hline & & $1960-70$ & $1970-80$ & $1980-90$ & $1990-2000$ & \\
\hline & Empleos Totales en Puerto Rico & 84,163 & 146,009 & 247,181 & 508,838 & \\
\hline & $\begin{array}{l}\text { Total Ocupaciones Ejecutivas, Administrativas } \\
\text { y Gerenciales para Puerto Rico }\end{array}$ & 12,742 & 18,725 & 35,426 & 25,327 & \\
\hline & $\begin{array}{l}\text { Total de Ocupaciones Ejecutivas, Administrativas y } \\
\text { Gerenciales en Recursos Humanos para Puerto Rico }\end{array}$ & 1,710 & 3,637 & 10,109 & $(2,527)$ & \\
\hline & Tasas de Crecimiento & & & & & \\
\hline & Empleos Totales en Puerto Rico & $1.4 \%$ & $2.0 \%$ & $2.7 \%$ & $4.0 \%$ & \\
\hline & $\begin{array}{l}\text { Total de Ocupaciones Ejecutivas, Administrativas y Gerenciales } \\
\text { para Puerto Rico }\end{array}$ & $3.4 \%$ & $3.5 \%$ & $4.5 \%$ & $2.3 \%$ & \\
\hline & \multicolumn{5}{|c|}{ Total Ocupaciones Ejecutivas, Administrativas y Gerenciales en Recursos Humanos para Puerto Rico } & $\begin{array}{l}20.0 \% \\
10.8 \% \\
10.8 \% \\
-1.7 \%\end{array}$ \\
\hline
\end{tabular}

Fuente: Departamento de Comercio Federal, Negociado del Censo, Censo de Población; 1960 al 2000 
Para el desarrollo del análisis debemos señalar que cada una de las ocupaciones se agrega a un subgrupo ocupacional y este subgrupo ocupacional se añade a uno de los grupos ocupacionales principales. En Puerto Rico existen sobre 1,600 ocupaciones que se agregan a 117 sub-grupos ocupaciones y estos últimos se añaden a en 13 grupos ocupacionales principales ${ }^{5}$. Un hallazgo que debemos señalar es que todas las ocupaciones relacionadas a la ocupación de recursos humanos se encuentran solamente en el primer grupo ocupacional de las ocupaciones ejecutivas, administrativas y gerenciales. La Tabla 9 muestra los empleos generados, la distribución porcentual y la tasa de crecimiento para el desglose de las ocupaciones en recursos humanos en Puerto Rico.

Si analizamos las tendencias en el empleo por género, según los datos publicados en los censos, observamos un total 12,959 empleos generados en las cuatro décadas que comprenden el periodo del 1960 al 2000; el aumento en empleo fue de 5,542 (42.9\%) empleos masculinos y 7,387 $(57.1 \%)$ empleos femeninos. El examen de las tasas de crecimiento desde 1960 hasta el 2000 muestra los resultados en forma más contundente. De 1960 al 2000 el empleo total para la ocupación de recursos humanos en Puerto Rico aumentó a una tasa anual promedio de $9.70 \%$, el empleo femenino a una tasa anual de $11.91 \%$, mientras que el empleo masculino solo creció a una tasa de $8.20 \%$. Esto datos reflejan la gran incursión del sexo femenino en la fuerza trabajadora empleada en el campo de los recursos humanos.

$\mathrm{Al}$ analizar el detalle de las ocupaciones de 1960 al 2000, la ocupación que generó la mayor cantidad de empleos fue la ocupación de los especialistas en personal, adiestramiento y relaciones laborales. Esta ocupación generó 9,129 empleos de los cuales 5,786 fueron empleos femeninos $(63.4 \%)$ con una tasa anual promedio de $5.7 \%$,

\footnotetext{
${ }^{5}$ Los grupos ocupacionales principales son los siguientes: I. Administradores y gerenciales, II. Profesionales, III. Ocupaciones de apoyo técnico, IV. Vendedores, V. Ocupaciones de apoyo administrativo, VI. Ocupaciones de servicios, VII. Ocupaciones agrícolas, VIII. Ocupaciones de reparadores y mecánicos, IX. Ocupaciones de trabajadores diestros de la construcción, X. Ocupaciones en trabajos de precisión, XI. Ocupaciones en armadores e inspectores, XII. Ocupaciones en operaciones de equipo de transportación y XIII. Ocupaciones en trabajadores de limpieza y Otros no diestros.
} 
Tabla 9

Tasa de crecimiento de la ocupación de recursos humanos en Puerto Rico

\begin{tabular}{|c|c|c|c|c|c|c|c|c|c|c|}
\hline & \multicolumn{3}{|c|}{$\begin{array}{l}\text { Empleos generados } \\
\text { desde } 1960 \text { al } 2000\end{array}$} & & \multicolumn{3}{|c|}{$\begin{array}{c}\text { Distribución porcentual } \\
1960 \text { al } 2000 \\
\end{array}$} & \multicolumn{3}{|c|}{$\begin{array}{l}\text { Tasa de crecimiento } \\
1960 \text { al } 2000\end{array}$} \\
\hline & Masculino & Femenino & Total & & Masculino & Femenino & Total & Masculino & Femenino & Total \\
\hline Grupo Ocupacional & & & & & & & & & & \\
\hline $\begin{array}{l}\text { I. Ocupaciones Ejecutivas, } \\
\text { Administrativas y Gerenciales }\end{array}$ & 38,936 & 53,284 & 92,220 & & $42.2 \%$ & $57.8 \%$ & $100.0 \%$ & 0.02 & 0.06 & 0.03 \\
\hline $\begin{array}{l}\text { Ocupaciones en Recursos } \\
\text { Humanos en Puerto Rico }\end{array}$ & 5,542 & 7,387 & 12,929 & & $42.9 \%$ & $57.1 \%$ & $100.0 \%$ & 0.08 & 0.12 & 0.10 \\
\hline $\begin{array}{l}\text { Otros Gerentes y } \\
\text { Administradores }\end{array}$ & 1,633 & 1,209 & 2,842 & $*$ & $57.5 \%$ & $42.5 \%$ & $100.0 \%$ & & & \\
\hline $\begin{array}{l}\text { Administrador de Personal y } \\
\text { Relaciones Laborales }\end{array}$ & 1,633 & 1,209 & 2,842 & $*$ & $57.5 \%$ & $42.5 \%$ & $100.0 \%$ & 0.05 & 0.07 & 0.06 \\
\hline $\begin{array}{l}\text { Relaciones Obrero-Patronal de } \\
\text { Personal }\end{array}$ & 3,343 & 5,786 & 9,129 & $*$ & $36.6 \%$ & $63.4 \%$ & $100.0 \%$ & & & \\
\hline $\begin{array}{l}\text { Especialistas en Personal, } \\
\text { Adiestramiento y Relaciones } \\
\text { Laborales }\end{array}$ & 3,343 & 5,786 & 9,129 & $*$ & $36.6 \%$ & $63.4 \%$ & $100.0 \%$ & 0.02 & 0.06 & 0.04 \\
\hline $\begin{array}{l}\text { Otras ocupaciones relacionadas } \\
\text { con la Gerencia }\end{array}$ & 566 & 392 & 958 & $*$ & $59.1 \%$ & $40.9 \%$ & $100.0 \%$ & 0.07 & 0.12 & 0.09 \\
\hline
\end{tabular}

Puerto Rico se comenzaron a reportar a partir de 1980 en los informes del Censo Federal de los Estados Unidos de América.

$\underset{\omega}{\infty}$ Fuente: Departamento de Comercio Federal, Negociado del Censo, Censo de Población; 1960 al 2000. 
mientras que el empleo masculino generó 3,343 (36.6\%) empleos y creció a una tasa de $1.60 \%$. Estos datos reflejan la gran incursión del sexo femenino en la fuerza trabajadora empleada en el campo de los recursos humanos.

Los estudios realizados sobre el análisis de la ocupación de los recursos humanos en la isla han sido escasos. Un análisis histórico de las personas empleadas en esta ocupación requiere de estudios postsecundarios. Se evidencia claramente cómo la participación del sexo femenino ha aumentado de forma acelerada en las ocupaciones relacionadas con los recursos humanos (Rivera Aponte, 2008; Ruiz Mercado, 1968). Del análisis se desprende que en Puerto Rico uno de los cambios fundamentales ocurridos en el campo de empleo es el marcado aumento del número de mujeres que han entrado al mercado de trabajo durante el periodo de 40 años analizado en este trabajo.

\section{BRECHA SALARIAL EN OCUPACIÓN DE RECURSOS HUMANOS}

El objetivo principal de esta sección es analizar las tendencias en el salario promedio por género y establecer si existe en Puerto Rico, al igual que en la mayoría de los países, una brecha salarial por género (gender wage gap). La fuente de datos usada son los censos de población de Puerto Rico.

Prácticamente en todos los países del mundo existe una diferencia entre el salario que se le paga a la mujer y el que devenga el hombre que ocupa el mismo puesto. Aunque en los últimos 40 años esta brecha se ha reducido, todavía persiste. Se han ofrecido varias explicaciones para este fenómeno, las cuales varían desde factores culturales hasta la discriminación. Por ejemplo, en los Estados Unidos, a pesar de que la brecha entre los salarios que reciben los hombres y los que reciben las mujeres se ha reducido sustancialmente, desde que se firmó el Acta de Paga Igual en 1963 (Equal Pay Act $)^{6}$, todavía existe una brecha significativa que no puede ser explicada por las diferencias en experiencias entre los trabajadores del sexo

\footnotetext{
${ }^{6}$ En 1963 el presidente Kennedy firmó el Equal Pay Act y lo convirtió en ley. De este modo, resulta ilegal pagar a hombres y mujeres empleados en el mismo establecimiento diferente salario en trabajos substancialmente similares.
} 
Análisis histórico de la profesión de reCursos humanos en Puerto Rico...

masculino y los del sexo femenino en el mercado laboral, y por las características en el tipo de trabajo que estos realizan. Después de mantenerse en alrededor de $60 \%$ desde la mitad de la década del 1950 al 1960, la proporción de la mediana de la paga recibida por la mujer a la recibida por el hombre (gender pay ratio), empezó a crecer en los últimos años de la década de 1960 al 1970 y alcanzó alrededor del $70 \%$ para el 1990. Ya para el 1997 el ratio había subido al $75 \%{ }^{7}$. No obstante, durante las últimas cuatro décadas la brecha salarial por género (BSG) se ha venido reduciendo. El aumento en la experiencia acumulada por las mujeres en el mercado de trabajo y su movimiento hacia ocupaciones de más alta paga, ha jugado un papel muy importante en el aumento en su salario promedio en relación con el del hombre. Los cambios en status familiar, en la estructura industrial y en las uniones sindicales también han sido factores que han contribuido a disminuir la BSG.

A pesar de la reducción en la brecha salarial, todavía su prevalencia permanece sin explicación. Muchos profesionales de esta disciplina atribuyen esta brecha a la discriminación contra el sexo femenino en el mercado de trabajo. La evidencia es que la discriminación en el mercado laboral persiste, a pesar de que es muy difícil determinar precisamente cuánta de la diferencia en el ratio de paga femenina a paga masculina se debe a discriminación y cuánto se debe a diferencias en las preferencias y la selección que hacen las mujeres y las que hacen los hombres (choices and preferences). La diferencia no explicada en la brecha puede ser una medida indirecta y un tanto burda de la mencionada brecha por discriminación. Algunos estudios han tratado de medir directamente este factor observando la diferencia de paga entre hombres y mujeres en tareas o trabajos similares o comparando la paga con medidas específicas de productividad. Estos estudios encuentran consistentemente evidencia de discriminación en el mercado laboral y favorecen la conclusión de que las mujeres todavía enfrentan trato diferencial en los trabajos que realizan.

\footnotetext{
${ }^{7}$ Council of Economic Advisers, Explaining trends in the gender wage gap, The White House, 1998.
} 
La discriminación por género en la fuerza laboral puede tomar varias formas, desde prácticas que reducen las oportunidades de que la mujer sea empleada, hasta diferencias de paga para hombres y mujeres que trabajan lado a lado haciendo el mismo trabajo e igualmente bien. Existe una variedad de teorías acerca de cómo y por qué la mujer enfrenta discriminación en el mercado laboral. Un determinado patrono puede no gustarle las empleadas del sexo femenino o subestimar sus habilidades; los clientes de determinado negocio o empresa pueden no gustarles las empleadas mujeres, así como también los compañeros trabajadores del sexo masculino pueden resentir y resistir el trabajar con mujeres. Estas actitudes pueden no estar dirigidas a todos los empleados del sexo femenino, sino a aquellos que ocupan puestos de alta jerarquía. Por ejemplo, los trabajadores del sexo masculino pueden no objetar el tener a mujeres trabajando para ellos pero sí pueden objetar cuando la mujer sea su superior. Además, los empleadores pueden enmarcarse en lo que se conoce como discriminación estadística, que quiere decir que ellos suponen que una mujer particular posee las características promedio de todas las mujeres. Por ejemplo, debido a que la mujer en promedio tiene más alta tasa de cambio en el trabajo (alta tasa de turnover) que el hombre, los empleadores pueden suponer que un determinado candidato del género femenino es más probable que deje la firma que un candidato del género masculino. La discriminación estadística, como otras formas de discriminación, es ilegal en prácticamente todos los países. A un patrono se le requiere que base la decisión de emplear a un trabajador y el pago que este devengará, sobre información específica acerca del individuo y no sobre presunciones basadas en género.

En el libro Mujer y brecha salarial: Reto del siglo XXI (Rivera Aponte, Ruiz Mercado \& García Toro, 2008), se presentó un análisis sobre la brecha en la isla. Este estudio demostró que sí existe una brecha salarial en Puerto Rico y que la misma está reduciéndose. Sin embargo, no se encontró qué causa la brecha salarial en Puerto Rico.

La Tabla 10 muestra el ingreso promedio por género y ocupación de recursos humanos para la década del 2000. La misma evidencia que para la ocupaciones de recursos humanos existe una brecha 
Tabla 10

Ingreso promedio por género y ocupación de recursos humanos, Puerto Rico, año 2000

\begin{tabular}{|l|c|c|c|c|c|}
\cline { 2 - 5 } \multicolumn{1}{c|}{} & Total & Masculino & Femenino & $\begin{array}{c}\text { Porciento del ingreso } \\
\text { promedio Femenino/ } \\
\text { Masculino }\end{array}$ & $\begin{array}{c}\text { Brecha } \\
\text { Salarial }\end{array}$ \\
\hline \multicolumn{1}{|c|}{ Grupo Ocupacional } & & & & & \\
\hline Demanda Total & & & & \\
\hline $\begin{array}{l}\text { I. Ocupaciones Ejecutivas, Administrativas y } \\
\text { Gerenciales }\end{array}$ & $\mathbf{2 8 , 5 7 5}$ & $\mathbf{3 0 , 3 5 2}$ & $\mathbf{2 4 , 6 7 8}$ & $\mathbf{0 . 8 1 3}$ & $\mathbf{0 . 1 8 7}$ \\
\hline $\begin{array}{l}\text { Administradores de Personal y Relaciones } \\
\text { Laborales }\end{array}$ & 25,397 & 28,522 & 21,175 & 0.742 & 0.258 \\
\hline Relaciones Obrero-Patronales de Personal & $\mathbf{2 0 , 0 8 3}$ & $\mathbf{2 3 , 4 9 6}$ & $\mathbf{1 7 , 9 9 6}$ & $\mathbf{0 . 7 6 6}$ & $\mathbf{0 . 2 3 4}$ \\
\hline $\begin{array}{l}\text { Especialistas en Personal, Adiestramiento y } \\
\text { Relaciones Laborales }\end{array}$ & 20,083 & 23,496 & 17,996 & 0.766 & 0.234 \\
\hline
\end{tabular}

Fuente: Department of Commerce. Census Bureau. Census of Population, Detailed Characteristics. Advance Query Systems, 2000. 
de salario que sobrepasa el $20.0 \%$. La diferencia en salarios es significativa todavía para el 2000.

Nos preguntamos lo siguiente: (1) ¿Las personas de la fuerza laboral que ejercen la ocupación de recursos humanos y trabajan en alguna oficina de recursos humanos, han investigado alguna variable que afecta este comportamiento de la brecha de salarial?, (2) ¿Conocían que existe una discrepancia significativa entre la ocupaciones relacionadas con el campo de los recursos humanos como unidad funcional dentro de la empresa? y (3) ¿Los cambios y las tendencias estructurales en Puerto Rico incrementarán o disminuirán más la diferencia salariales por género?

\section{ReCOMENDACIONES y CONCLUSIONES}

En esta investigación se ha analizado el cambio de la ocupación de la profesión de los recursos humanos como disciplina por género y salarios. Se ha dividido el estudio en tres secciones: (1) Educación y recursos humanos, (2) Tendencias generales en el empleo de la profesión relacionada con el campo de los recursos humanos y el género, y (3) Determinación del salario promedio para la ocupación de los recursos humanos en Puerto Rico para el 2000.

En Puerto Rico, en el 2006, se realizó un estudio sobre la planificación de los recursos humanos en la isla (Rivera Aponte, 2006). En el caso específico de la profesión de recursos humanos como disciplina, son escasos los estudios sobre los cambios en la estructura del empleo por género enmarcado en el proceso de planificación de los recursos humanos. Asimismo, no se ha realizado una investigación histórica detallada concerniente a los cambios ocupacionales experimentados en el mercado laboral que comprenda un período de 40 años o más en la ocupación del campo profesional de los recursos humanos como disciplina. Además, no se han presentado investigaciones relacionadas con la cantidad de programas que se ofrecen a nivel graduado y subgraduado en esta disciplina académica de recursos humanos en administración de empresas.

En Puerto Rico unas diez instituciones de educación superior ofrecen 53 programas académicos específicamente en la disciplina 
de Recursos Humanos en Administración de Empresas. La fuerza laboral que ejerce la ocupación de recursos humanos en la isla requiere estudios postsecundarios. Encontramos que la fuerza laboral que ejerce la ocupación como profesión de recursos humanos se ubica en el primer grupo ocupacional principal de administradores, ejecutivos y gerentes. Al estar dentro de este grupo ocupacional principal, en promedio tienden a tener los salarios más altos dentro de la estructura ocupacional. Se evidenció que desde el 1960 al 2000, la tasa de crecimiento anual aumentó en $9.7 \%$; específicamente, el aumento se produjo en el género femenino (11.91\%), mientras que la tasa de crecimiento del género masculino fue de crecimiento de $8.22 \%$. Se encontró, además, que todavía existe una brecha de salario de $23.5 \%$ en contra del género femenino en la ocupación de los recursos humanos. Se evidenció también un aumento en la participación del género femenino en las ocupaciones de recursos humanos desde el 1960 al 2000.

Este estudio demostró que existe un excedente en ofrecimientos académicos a nivel postsecundario en Puerto Rico para esta disciplina; que existe una brecha salarial en este campo de estudio en Puerto Rico y que la misma ha estado reduciéndose en el transcurso de las últimas décadas. Sin embargo, los estudios realizados no han conseguido determinar las causas sobre la persistencia de esta brecha salarial en Puerto Rico.

Se recomienda continuar realizando investigaciones académicas sobre el comportamiento y la tendencia en el mercado laboral de las ocupaciones relacionadas con la profesión de los recursos humanos en Puerto Rico. Se recomienda, además, realizar un estudio más exhaustivo sobre la integración entre la oferta y la demanda del mercado laboral en Puerto Rico por ocupación de esta profesión. La falta de planificación de recursos humanos ha generado un excedente de programas académicos en esta disciplina. Ante los escenarios de crisis estructural que experimenta Puerto Rico, el análisis crítico de la política pública deberá generar conciencia sobre los excedentes de ofrecimientos académicos y la reducción de las oportunidades de empleos en el campo de los recursos humanos en la isla. Se sugiere generar una estrategia para que se pueda desagregar más la ocupación de los recursos humanos en 
Análisis histórico de la profesión de reCursos humanos en Puerto Rico...

las siguientes categorías ocupacionales: selección y reclutamiento, adiestramientos, compensación y salarios, seguridad ocupacional y relaciones laborales, entre otras.

Se aconseja realizar estudios por áreas regionales y por los respectivos sectores industriales en Puerto Rico para examinar las tendencias de los mercados laborales vinculados a los recursos humanos. Se recomienda realizar análisis comparativos de los territorios de los Estados Unidos de América frente a Puerto Rico, para comparar las tendencias de participación en el mercado laboral, nivel de escolaridad, género y brecha de salario, entre otras, correspondientes a dichas ocupaciones en recursos humanos. 


\section{Referencias}

Barney, J. B. (2001). Resource-base theories of competitive advantage: A ten-year retrospective on the resource-based view. Journal of Management, 27, 643-650.

Chiavetano, I. (1995). Introducción a la teoría general de la administración (4ta ed.). México: Mc Graw Hill.

Daniels, K., Davis, A., Harris, A., Parkes, C., Scully, J., Shipton, H. \& West, M. (2008). Strategic human resource management. Australia and New Zealand: Mc Graw Hill.

Director, S. M. (1985). Strategic planning for human resource. Work in American institute studies in productivity. New York: Pergamon Press.

Haines III, V., Brouillard, S. \& Cadieux, N. (2010). Une analyse longitudinale (1975-2005) de l'évolution de la profession ressources humaines. Relations Industrielles, 65, 491.

Haslinda, A. (2009). Evolving terms of human resource management and development. Uluslacarast sosyal Arasturmadar Dergisi. The Journal of International Social Research, 2(9), 180-186.

Hopkins, M. J. (1988). Employment forecasting: The employment problem in industrialized countries. London: International Labor Organization.

Horrigan, M. (February 2004). Employment projections to 2012: Concepts and context. Monthly Labor Review, 127, 3.

International Labor Office. (2004). Global Employment Trends. January. United States of America.

Khandwalla, P. (1977). The design of organizations. New York: Horcourt Brace Jovanovich, Inc.

Leontief, W. (1953). The structure of the American economy - 1919-1939. New York: Oxford University Press.

Malik, N. (2009, 18 de febrero). Academic Leadership. The Online Journal. Recuperado de http://www.academicleadership.org/ article/emergence-of-strategic-human-resouce-managementhistorical-perspective 
Análisis histórico de LA profesión de RECURSOS humanos en Puerto Rico...

Mora, E. I. (2011). El periodo moderno de la economía de Puerto Rico. En E. I. Mora (Ed.), Economía de Puerto Rico (pp. 53-61). México: Mc Graw Hill Interamericana Editores.

Oficina del Censo. (1950). Negociado del Censo Federal: Censo de Población de Puerto Rico. Junta de Planificación. Programa de Planificación Económica y Social. San Juan: Departamento de Comercio de los Estados Unidos de América.

Oficina del Censo. (1960). Negociado del Censo Federal: Censo de Población de Puerto Rico. Junta de Planificación. Programa de Planificación Económica y Social. San Juan: Departamento de Comercio de los Estados Unidos de América.

Oficina del Censo. (1970). Negociado del Censo Federal: Censo de Población de Puerto Rico. Junta de Planificación. Programa de Planificación Económica y Social. San Juan: Departamento de Comercio de los Estados Unidos de América.

Oficina del Censo. (1980). Negociado del Censo Federal: Censo de Población de Puerto Rico. Junta de Planificación. Programa de Planificación Económica y Social. San Juan: Departamento de Comercio de los Estados Unidos de América.

Oficina del Censo. (1990). Negociado del Censo Federal: Censo de Población de Puerto Rico. Junta de Planificación. Programa de Planificación Económica y Social. San Juan: Departamento de Comercio de los Estados Unidos de América.

Oficina del Censo. (2000). Negociado del Censo Federal: Censo de Población de Puerto Rico. Junta de Planificación. Programa de Planificación Económica y Social. San Juan: Departamento de Comercio de los Estados Unidos de América.

Ricardo, D. (1817). On the principle of political economic and taxaction. New York: E. P. Dutton. Citado en Barney, J. B. (2001). Resource-based theories of competitive advantage: A ten-year retrospective on the resource-based view. Journal of Management, 27(6), 643-650.

Rivera Aponte, A. L., Ruiz Mercado, A. L. \& García Toro, V. I. (2007). Mujer y brecha salarial: Reto del siglo XXI. San Juan: Oficina de la Procuradora de las Mujeres. 
Rivera Aponte, A. L. (2006). Cambios en la estructura industrial y ocupacional del empleo en el proceso de desarrollo económico de Puerto Rico: Un análisis histórico de la planificación de los recursos humanos usando el modelo de insumo-producto del 1960 al 2000. (Tesis inédita de doctorado). Universidad Interamericana de Puerto Rico, Puerto Rico.

Roca de Torres, I., Gaztambide Géigel, A. \& Morales Vázquez, M. (2003). La Facultad de Ciencias Sociales: Testimonios de medio siglo, 1943-1993. Universidad de Puerto Rico, Facultad de Ciencias Sociales. San Juan: Publicaciones Puertorriqueñas.

Rudman, R. (2010). Human resource management in New Zealand (5th ed.). New Zealand: Pearson.

Ruíz Acevedo, A. (1996). La glorificación y crucifixión del sistema de mérito en Puerto Rico. Revista Administración Pública, 29(1), 13-28.

Ruíz Mercado, A. L. (1980, 18 de junio). Transformación estructural en el sector de los servicios de la economía de Puerto Rico. Cambios en el patrón de empleo femenino por el sector industrial y por ocupación durante el proceso de desarrollo económico en Puerto Rico. Ensayos y Monografías, Unidad de Investigación de Ciencias Sociales, Departamento de Economía, Núm. 18, 1-22. Recuperado de http://economia.uprrp.edu/ ensayomono.html

Ruíz Mercado, A. L. (1986). Requerimiento de recursos humanos por ocupación y sector industrial, años 1984-1990. San Juan: Puerto Rico Occupational Information Coordinating Committee.

Ruíz Mercado, A. L. (1990). Aplicaciones del modelo de insumo producto a la economía de Puerto Rico. Ensayos y Monografías, Unidad de Investigación de Ciencias Sociales, Departamento de Economía, Núm. 55, 1-17. Recuperado de http:/ / economia. uprrp.edu/ensayomono.html

Safa, H. I. (1998). De mantenidas a proveedoras: mujeres e industrialización en el Caribe. San Juan: Editorial de la Universidad de Puerto Rico.

Santana Rabell, L. (1993). Fulgor y decadencia de la Administración Pública en Puerto Rico: Agenda para la reforma. Revista de Administración Pública, 25, 161-182. 
Análisis histórico de la profesión de reCursos humanos en Puerto Rico...

Schermershorn, J. R. (2008). Human resource management. New Jersey: John Wiley \& Sons, Inc.

Standing, G. (1989). Global feminization through flexible labor. World Development 17(7), 1077-1096.

Sukumar, S. (2011). Difference between human resource management and personnel management. Recuperado de http:// www.buzzle.com/articles/difference-between-human-resourcemanagement-and-personnel-management.html

Taylor, F. (1911). The principle of scientific management. New York: Harper \& Bros.

The Chartered Institute of Personnel and Developmente. (2011). History of HR and the CIPD. The Chartered Institute of Personnel and Development CIPD. Recuperado de http://www.cipd.co.uk/ hr-resources/factsheets/history-hr-cipd.aspx 